\title{
EVALUATING OPERATING DISTRIBUTION STRATEGIES IN SUPPLY CHAIN MANAGEMENT USING A HYBRID SIMULATION APPROACH
}

\author{
Tarak A. Housein* Amer A Boushaala** \\ Department of Industrial and Manufacturing Systems Engineering, \\ University of Garyounis - LIBYA \\ E-mails:*tshatshat@yahoo.com,**boushaala@yahoo.com
}

\begin{abstract}
This paper presents an innovative hybrid simulation modeling approach developed for supply chains. The model approach has been built to investigate different inventory policies and transportation strategies in a supply chain network that minimize the system wide cost. The complex system is modelled using the powerful discrete-event simulation software DOSIMIS-3 and C++ DLL is developed for this purpose. This Hybrid approach combines the analytical inventory models, heuristics transportation strategies approaches, and simulation models to model different network structures and operating distribution strategies in supply chains.

The developed approach is described in detail for the analysis of supply chains. The results from experiments that demonstrate the benefits of the proposed architecture are discussed and presented.

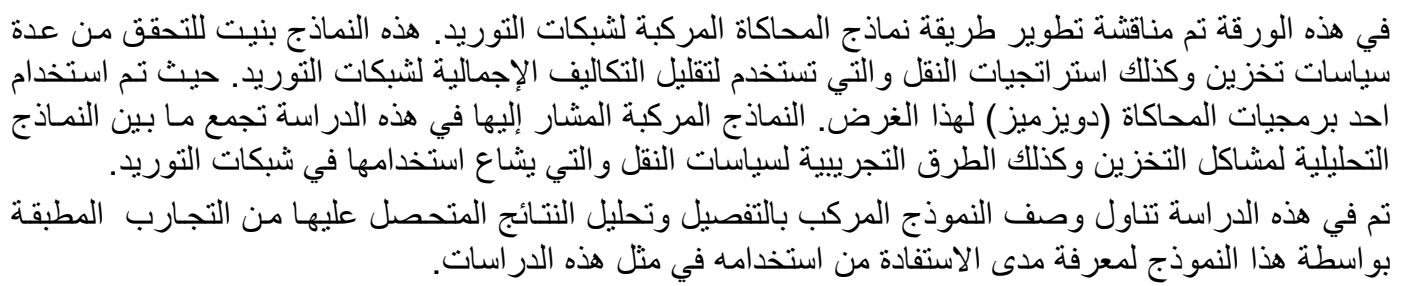

Keywords: Supply chain management, Discrete-event simulation, Inventory policies, Transportation strategies.

\section{INTRODUCTION}

A supply chain is a network of facilities that performs the functions of procurement of materials, transformation of these materials into intermediate and finished products, and the distribution of these finished products to customers. A typical supply chain may consist of many participants, such as suppliers, manufacturers, distributors, retailers and the end customer. Each participant in the supply chain has his own set of objectives. Due to its inherent complexity, analytical modeling of supply chains becomes difficult. Moreover, a typical supply chain faces uncertainty in many of its activities, for example, in the supply of raw materials from the suppliers. Under such complex and uncertain situations, simulation becomes the best alternative for the analysis.

In recent years, operation research models have put more emphasis on modeling and analyzing multiechelon distribution systems. Though Supply Chain Management (SCM) is relatively new, while the idea of coordination is old.
The main drawback inmost analytical models is the fact that numerous constraints have to be fulfilled before results can be applied in practice. Most of the models only take only few variables into account, such as inventory and running out of stock, and ignore other costs, such as, order processing, handling and transportation. Furthermore, these models neglect capacity restrictions.

Computer simulation is, therefore, an especially effective tool to help investigate and analyze complex problems since it can be applied to operational problems that are too difficult to be modeled analytically. Moreover, mathematical models require too many simplifications and assumptions to model operating strategies in realistic supply chain problems. Discrete event simulation permits complex logistics systems to be modeled more realistically.

Two major approaches have been used to model and to design the supply chain problems. The first one focuses on very accurate mathematical descriptions of cost functions named as mathematical optimization techniques that can be classified into 
two branches. The two branches they are exact algorithms which guarantee to find optimal solutions minimizing cost functions or they are heuristic algorithms which find good but not necessarily optimal solutions. The second approach is the simulation models that provide a mechanism to evaluate specified design alternatives created by the designer.

The supply chain network that we would like to analyze and investigate is typically complex. It considers many strategies changed over time. The mathematical optimization techniques have some limitation as they deal with static models. Simulation based tools, however, are capable of characterizing system performance for a given design while taking the dynamics of the system into account. Therefore a hybrid approach that combines analytical, heuristics, and simulation models is needed. This efficient approach is capable to model and evaluate the different network structures and operating distribution strategies in supply chain management.

The purpose of this paper is, therefore, to present a hybrid simulation model approach of a complex supply chain network used to evaluate and improve the operating distribution strategies with impact on different distribution (Inventory \&Transportation) strategies. This approach is modeled with a discrete event simulation software linked with $\mathrm{C}++$ a program which has been developed for this purpose. Simulation results have been summarized and discussed.

To evaluate the effectiveness of the supply chains, a number of performance measures have been modeled. Some of these measures are the Logistics Cost and Customer satisfy.

Many experiments and analysis have been implemented by this approach. These analyses prove that, a hybrid simulation approach is a useful tool for studying and evaluating supply chain management.

Finally conclusions and suggestions for future research have been presented.

\section{LITERATURE REVIEW}

A large body of literature exists on the supply chain modeling approaches. Two of the most common ways of analyzing a supply chain are simulation and analytical modeling. Thomas and Griffin [1] defined three categories of the operational coordination of the supply chain management. These categories are: (1) Buyer-vendor coordination, (2) Production-distribution coordination, (3) Inventorydistribution coordination. Also they have made critical review study on the supply chain coordination and list some topics for future work.

In supply chain modeling, effort is made to consider the effect of policies on the performance of the supply chain. The effects of policies are tested either analytically or through simulation. In the case of simulation of supply chains, effort involved in building the supply chain simulation model can be reduced to a great extent if the models can be built hierarchically from existing modules. Eliter et al. [2] worked on the concept of Agent Programs. An agent consists of a body of software code that supports a well-defined application programmer interface and a semantic wrapper that contains a wealth of information. As part of the work, the team developed agents for various functions of supply chain management systems. A simulation model of a supply chain application based on agents was built using commercial software such as Microsoft Access and ESRI's MapObject. Swaminathan et al. [3] described a supply chain modeling framework that can be used for constructing supply chain simulation models. They develop software components for representing various types of supply chain agents such as retailers, manufacturers and transporters. The authors divide the set of elements in their supply chain library into two categories: Structural Elements and Control Elements. Structural elements correspond to agents (eg manufacturer agents, transportation agents) and control elements correspond to the control policies.

Lee and Kim [4] proposed a hybrid approach which is a specific problem solving procedure combining analytic and simulation methods to solve production-distribution problems in supply chains.

Jain et al. [5] observed that the level of details included in the development of a simulation model should be appropriate to the objective of the study. They concluded that inclusion of more detail than necessary can easily lead to a large an effort for the objective at hand and the effort not being approved by the parent organization. They developed a high level supply chain simulation model using a generalpurpose simulation model. Their justification for using general-purpose simulation software instead of a commercially available supply chain simulation tool was that general-purpose simulation software lets the user select the desired level of abstraction. IBM Supply Chain Simulator is one of the commercially available packages for simulating supply chains.

Díaz and Buxmann [6] examined how the benefits of Supply Chain Management, as reported by the literature and widely accepted, can be proved using simulation. They presented selected results of a survey conducted on the European automotive industry, which show an evident needed for transparency, in terms of the quantification of the added-value of Supply Chain Management. For this purpose an XML-based prototype for modeling and simulating cooperative scenarios in supply chains is 
introduced, and illustrated its flexible architecture and the interaction between modeled scenarios and optimization routines through XML interfaces. In the context of this prototype, the authors described a simulation scenario in which the transportation activities in a supply chain are modeled and planned.

Chu [7] provided a comprehensive listing of simulation studies to summarize their findings and to identify future research. He classified the simulation studies on supply chains based on its major focus.

Terzi and Cavalieri [8] presented a comprehensive review made on more than 80 articles, the main purpose being to ascertain which general objectives simulation is generally called to solve and which paradigms and simulation tools are most suitable. Useful prescriptions, both for practitioners and researchers, on the applicability of simulation to decision-making processes within the supply chain context were also derived.

By providing a systematic quantitative and objective evaluation of the outcomes resulting from different possible planning scenarios, from demand planning to transportation and distribution planning, simulation techniques can make companies more aware of the benefits coming out from integrated and co-operating strategies with their upstream/downstream locations rather than following myopically an antagonistic behavior with them [8].

Kleijnen [9] surveyed four types of simulation, and discussed four methodological issues. These four simulation types are spreadsheets, system dynamics (SD), discrete-event dynamic systems simulation (DEDS) and business games. He explained how different types can answer different questions in SCM.

Referring to the classifications and suggestions for future researches in the literature, the developed hybrid simulation approach in this paper can be considered a new simulation study to an industry, and new enhancement strategies are to be tested and combined.

\section{DESCRIPTION OF A HYBRID SIMULATION MODELING APPROACH}

The hybrid simulation approach consists of integrating analytical inventory models, heuristics distribution (Transportation) strategies, and simulation model of the total system, developing their solution methods, and using their solution methods together for problem solving and evaluating the supply chain performances.

The procedure of the hybrid simulation approach is described by Fig. 1 .

General-purpose discrete event simulation software cannot be directly used for simulating supply chains. The simulation modules provided in the software should be combined or modified to represent the activities of supply chains [10]. Based on this idea, a hybrid simulation modeling approach [HSMA] is developed. The HSMA is a discrete event model, developed based on the combining of the eventscheduling and activity-scanning approaches [11]. The HSMA was created using DOSIMIS-3, with support of a C++ program (DLL). It was developed specifically for the supply chain simulation purpose. Some other tools were customized for the use with this particular tool. The database tools used in HSMA interface utilized the widely available application programs, such as Microsoft Excel, Access and Visual Basic for Applications (VBA), to assist in scrutinizing the data.

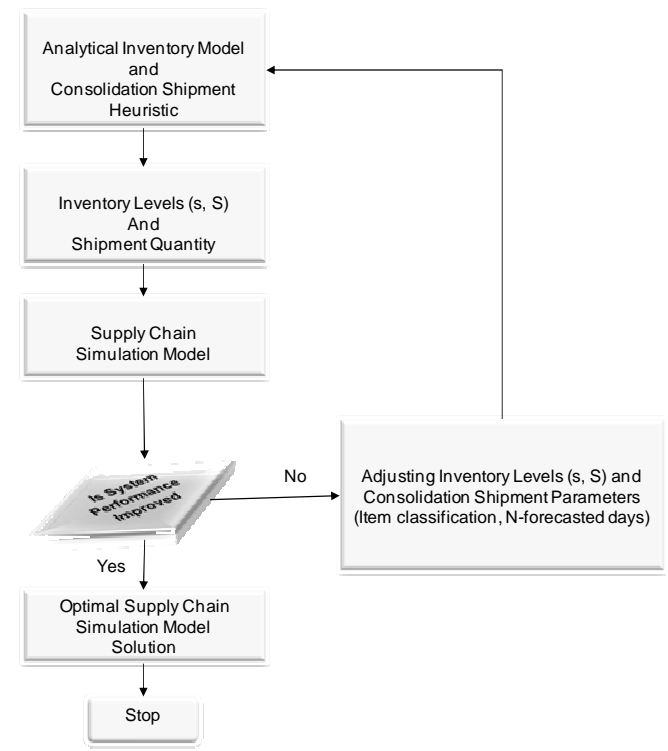

Fig. 1 Hybrid solution procedure

DOSIMIS-3 is a discrete event simulation tool developed by SDZ GmbH [12]. It is an interactive using objects and graphical working with Windows 95/98/NT/XP. The package is modular-oriented. The simulator works event-discrete and allows simulation of time-discrete material flow systems. A simulated production process can be developed graphically on the screen. Standard elements, such as sources, sinks, work stations, storages, vehicles etc., which in their structure represent essential modules from the logistics field, allow a rational layout by means of a menu-controlled user interface. Modules with several entrances and exits deploy an intelligence over which local strategies, such as FIFO, minimal occupation of the succeeding module etc. can be realized when controlling the object flow.

The DOSIMIS-3 is controlled by the Dynamic Link Library (DLL) called location controller programmed by visual $\mathrm{C}++$ program under the DOSIMIS-3 simulation platform to construct the 
HSMA. The interaction between DOSIMIS-3, DLL, and other tools is presented in Fig. 2.

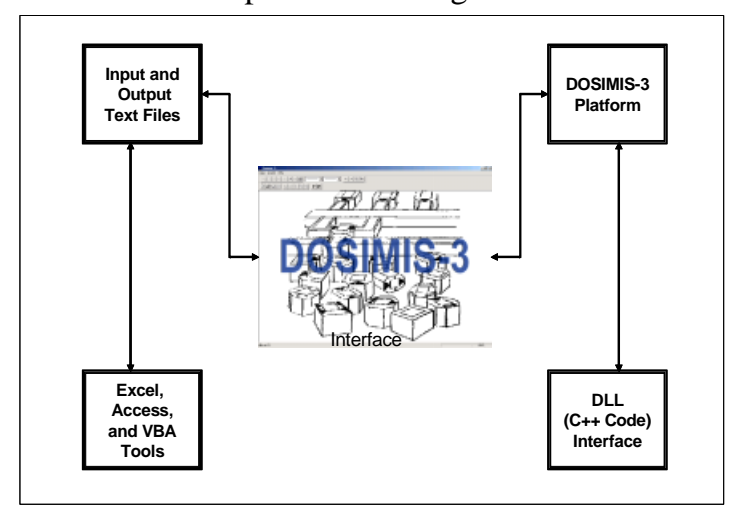

Fig. 2 DOSIMIS-3, DLL and other tools interaction

The HSMA consists of the basic supply chain distribution network elements representing all the locations, items (materials and resources), inventories, and customers in the network. The user enters or imports data about the supply chain distribution network, and the simulation tool predicts the performance of the proposed network, operationally and financially. If the existing network is entered in, alternative scenarios can be tried, in order to see how the existing operation would function if, for example, demand falls, rises or spikes seasonally, for one product, several products, or entire product classes.

The HSMA also allows the user to try out changes in the existing distribution network configuration, to see what the impact would be. Thus, users can evaluate what the effect on the financial status of a previous scenario would have been on if they had implemented centralization via decentralization, or transshipment points via tradition centre distribution.

The following network data is needed to implement the HSMA:

\section{Network Structure:}

- Items - weight, volume, source.

- Location - type, name, number.

- Customer Order (Demand) - date, type and location.

- Network Policies and Strategies:

- Inventory Policy - determines if (at all) the inventory is stocked or not, how often it is counted, when is it replenished, carrying costs,

- Replenishment Policy - determines the replenishment order size and based on what concept (Only Demanded Order replenishment, others ),
- Sourcing Strategy - determines the source (location) of satisfaction of the customer order (order type, location, item type and number).

- Transportation Strategy - determines how are shipments transported (Less than Truck Load (LTL), Full Truck Load (FTL) and how much do shipments cost.

\section{Production:}

- Productions are modeled using the black box: simple production lead time and quantities.

- The plants have an unlimited capacity to supply any item or product.

\subsection{Inventory Analytical Models Product}

In HSMA, the location inventory in the supply chain network uses the continuous review installation stock $(\mathrm{s}, \mathrm{S})$ policy where $\mathrm{s}$ is the reorder point and $\mathrm{S}$ is the order up-to-level. The stocks are controlled in a decentralized manner by installation stock re-order points [13]. The HSMA checks continuously whether the customer orders have reduced the net current inventory position of any item at each location to a level less than the reorder point (si). When the net current inventory position falls below the reorder point, the inventory system will make a replenishment order from the inventory of its upstream location. The process continues until enough material has been received at the location to fulfill all current orders. Net current inventory position is a number calculated throughout the simulation.

However, if the stock on-hand cannot fulfill the order, the order will be held in a queue in the inventory system; this means the order becomes a backorder. The backorders are held in a queue in the location. The backorder queue is implemented as a first-in-first-out (FIFO) queue. Whenever the onhand, on-order, and backlog are updated, the net current inventory position (inventory on-hand + onorder - backorders) will be updated. The inventory system can fullfill the backorders only when the replenishment orders (shipments) arrive. There is a time delay for the shipments to arrive (lead time).

During the simulation, the HSMA generates Inventory Tracking files that keep track of the possible status of an existing orders that are open or delivered, and the inventory status include received, in-process, in-transit, or delivered.

The values of $\mathrm{s}$ and $\mathrm{S}$ parameters for each item type are estimated by different ways as presented in inventory theories to see the effect of selecting these control parameters on the performance of distribution strategies and also to see the benefit of using the hybrid simulation as a tool for estimating the optimal value of such parameters. 


\subsection{Transportation Strategies}

In this tool all items are placed on pallets for shipment. Trucks are used to carry the shipments. Direct shipment between locations is considered and no routing decisions have been taken. Two common shipping strategies have been modelled. They are the Full Truckload (FTL) and the Less-than-Truckload (LTL) strategies. The planner can select one of them through the appropriate selection of the replenishment policy.

Transportation strategies include the application of different types of shipment consolidation (freight consolidation) policies. The consolidation policy coordinates the shipping of different item orders for the same destination, and this can lead to a reduction in transportation costs. Based on that two heuristic, consolidation concepts are developed and programmed to integrate into the HSMA. The two consideration are:

1. Item classification consolidation concept.

2. N-days forecasted demand concept.

In the first heuristic, the construction of consolidated shipment is designed based on the item classification like ABC-Classification. For example if the shipment size is less than the truck capacity, the rest of truck capacity is filled by A-Items. In this heuristic, there is guarantee that the shipment quantities will generate a full truckload.

In the second heuristic, the construction of consolidated shipment is designed based on forecasted demand for the next N-days. For example if the shipment size is less than the truck capacity, the rest of truck capacity is filled by the selected number of next forecasted demand days of each items.

Forecasting techniques are used to forecast a demand in advance.

\section{SUPPLY CHAIN CASE STUDY}

In this Paper, a real life distribution supply chain in a food industry has been considered. The company is located in a European country, it produces three major brands of products, and holds more than 3000 different SKUs per day (stock keeping unit).

Currently, this company has several production locations (plants), central warehouses, about 30 regional distribution centers, and approximately more than 5000 retailers and customers spread over the country. The warehouses are located at the plants and deliver different items to distribution centers which in turn supply a large set of geographically scattered retailers. It is assumed that the warehouses replenish multi-items from infinite supply plants and act merely as coordinators of the supply process, but do not hold any stocks.

The distribution centers hold stocks of multiitems and use common inventory policies to replenish their inventory levels. Each distribution centre faces a dynamic demand rate.

The logistics manager in the company had to study some operating dustribution strategies to evaluate and improve the existing network. The hybrid simulation tool was implemented to investigate these strategies.

\subsection{Simulation Assumptions}

The HSMA was implemented under the following assumptions:

- Standard European Pallet (SEP) with a height of $2.4 \mathrm{~m}$ height will be used to move the full pallet product from the warehouses to regional distribution centre.

- Length $\mathrm{x}$ Width $\mathrm{x}$ Height $(\mathrm{LxWxH})=1.2 \mathrm{~m} \times$ $0.8 \mathrm{~m} \times 2.4 \mathrm{~m}$

- The mixed pallet of the following dimensions will be used to move the product from the regional distribution centre to retailers and customers :

$\mathrm{L} \times \mathrm{W} \times \mathrm{H}=1.2 \mathrm{~m} \times 0.8 \mathrm{~m} \times 1.8 \mathrm{~m}$. Percentage of utilized space: $70 \%$

- Transportation costs based on the direct tour with one destination will be accounted, no routing allowed.

- Direct shipments from central warehouse to retailers or customers are allowed if the shipment size is up to truck capacity.

- The used truck capacity is 38 SEP

- All transportations between locations are done by Third Party Logistics (3PL) providers. Therefore the fixed transportation costs are not considered.

- The customer orders are satisfied and delivered completely (all order lines) based on a first-infirst-out (FIFO) rule.

\subsection{Model Input Data}

A significant amount of historic data from the company's ERP system has been used to conduct the simulation study. In the first phase of the simulation study, an extensive data analysis was accomplished.

For example, information which covers the following customer attributes was considered in the simulation model:

- Customer name

- Customer number

- Address

- Travel Distance from the regional distribution centers

- Type of customer

For the computation of the transport costs, a detailed transport cost matrix from the company has been considered for the calculation based on different calculation criteria. The other costs which were 
considered in the simulation study are summarized in the next section.

\section{Logistics Costs}

The logistics costs can be classified as follows:

- Transportation costs : Depends on number of pallets and distance (ZIP code)

- Storage and handling costs (inventory costs)

i. Monthly storage costs

ii. Order costs

iii. Order picking costs per pallet

iv. Warehouse outgoing goods costs per pallet

v. Regional distribution centers incoming goods costs per pallet

- $\quad$ Toll fees

Inbound toll costs (warehouse to distribution centers) per kilometer traveled.

Another basic rule in assigning products to storage locations is taking into consideration the dimensions. Cube matching of the items with the storage locations is essential to eliminate space inefficiencies. Shelf dimensions should be spacious enough to allow easy picking, but tight enough to avoid unused space. Here is a bad usage of shelf space versus good usage.

\subsection{Model Output Reports}

The key to the usefulness of a supply chain simulator is the ability to translate the simulation information into costs and financial reports (Bagchi 1998). This is typically achieved through the use of activity-based costing models. Therefore, the HSMA generates more detailed information about the activity-based costs by each location in the network. Once the model has been simulated, the user can get detailed statistics on almost every aspect of the model's performance. The data from the simulation has been summarized in a number of different ways to provide information about the following areas:

- $\quad$ Service Level (Fill rate)

- Transportation

- Inventory

- Activity -based Costs

Some of the standard output reports obtained by the HSMA: financial summary reports, inventory levels, transportation rate/levels, and service order degree reports.

\section{SIMULATION EXPERIMENT \& RESULTS}

In this study, six scenarios are designed.

\subsection{Experiment Design}

The design of each experiment strategy is constructed mainly based on the following two inventory control parameters and one consolidation parameter:

1. Reorder point (fixed for each class or individual based on service level, s).

2. Maximum inventory level (order up-to-level, S).

3. ABC Item classification

4. N-days forecasted demand.

For designing these experiments, some assumptions are considered:

- In all experiments the beginning inventory is assumed to equal the reorder point and the lead time is four days (LT = 4 Days).

- The continuous review installation stock $(\mathrm{s}, \mathrm{S})$ policy where $\mathrm{s}$ is the reorder point and $\mathrm{S}$ is the order up-to-level is used as inventory policy for all the experiments.

- The physical maximum capacity of inventory levels should be limited.

- The customer orders are filled completely.

- The filling of customer orders is based on a firstin-first-out (FIFO) rule.

- The number of distribution centers that have been considered is twenty four (DC's $=24$ ).

The reorder point (s) can be calculated based on Cycle Service Level (CSL) procedure (Chopra 2004).

Cycle Service Level (CSL) is the fraction of replenishment cycles that end with all the customer demand being met. A replenishment cycle is the interval between two successive replenishment deliveries. Therefore, CSL is equal to the probability of not having a stockout in a replenishment cycle. In this case safety stock (safety inventory) is determined based on desired Cycle Service Level (CSL) of decision makers. The reorder point (s) for each item will be calculated based on this result.

The procedure can be described as follows:

Observe that a stockout occurs in a cycle if demand during the lead time is larger than the ROP (s). Thus, identify the safety inventory $S S$ such that the following is true:

$\mathrm{CSL}=$ probability (demand during lead time $\leq$ $D_{L}+S S$ )

If demand during lead time is normally distributed with a mean of $D_{L}$ and a standard deviation of $\sigma_{L}$ where,

$$
D_{L}=D \times L \text { and } \sigma_{L}=\sqrt{L} \times \sigma_{D},
$$

so that

$$
F\left(D_{L}+S S, D_{L}, \sigma_{L}\right)=C S L
$$


Tarak A. Housein, Amer A Boushaala, "Evaluating Operating Distribution Strategies in Supply Chain ..."

By using the definition of the inverse normal, the equation can be derived

$$
\begin{aligned}
& D_{L}+S S=F^{-1}\left(C S L, D_{L}, \sigma_{L}\right), \text { or } \\
& S S=F^{-1}\left(C S L, D_{L}, \sigma_{L}\right)-D_{L}
\end{aligned}
$$

By using the definition of standard normal distribution, its inverse can be modified as follows:

$$
S S=F_{S}^{-1}(C S L) \times \sigma_{L}
$$

Finally, the reorder point (s) can be calculated by

$$
s=D_{L}+S S
$$

where:

$C S L=$ Desired cycle service level,

$D=$ Average demand,

$\sigma_{D}=$ Standard deviation of demand,

$D_{L}=$ Mean demand during lead time,

$\sigma_{L}=$ Standard deviation of demand during lead time,

$L=$ Average lead time for replenishment

$S S=$ Safety stock

$s=$ Reorder point

The maximum inventory level of each item class is calculated by the following equation:

$\mathrm{S}=$ Number of Days $*$ Average Demand (Daily)

The design of all the six scenarios are summarized in the following two tables:

\begin{tabular}{|c|c|c|c|c|c|c|c|}
\hline \multicolumn{2}{|c|}{$\begin{array}{c}\text { Operating Distribution } \\
\text { Strategy }\end{array}$} & \multirow{2}{*}{\begin{tabular}{|l|}
$\mathrm{S} 1$ \\
$\mathrm{X}$
\end{tabular}} & \multirow{2}{*}{$\begin{array}{l}\mathrm{S} 2 \\
\mathrm{X}\end{array}$} & \multirow{2}{*}{\begin{tabular}{l|}
$\mathrm{S} 3$ \\
$\mathrm{X}$
\end{tabular}} & \multirow[t]{2}{*}{$\mathrm{S} 4$} & \multirow[t]{2}{*}{ S5 } & \multirow[t]{2}{*}{ S6 } \\
\hline$\stackrel{\overrightarrow{0}}{0}$ & CLS $=80 \%$ & & & & & & \\
\hline$\stackrel{0}{\Xi}$ & CLS $=90 \%$ & & & & $\mathrm{X}$ & $\mathrm{X}$ & $\mathrm{X}$ \\
\hline \multirow{3}{*}{ 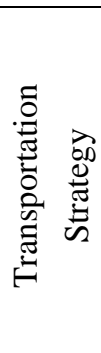 } & $\begin{array}{c}\text { LTL Concept } \\
\text { (Only Demanded } \\
\text { Order) }\end{array}$ & $\mathrm{X}$ & & & $\mathrm{X}$ & & \\
\hline & $\begin{array}{c}\text { FTL Concept } \\
\text { (N-Days=2 days) }\end{array}$ & & $\mathrm{X}$ & & & $\mathrm{X}$ & \\
\hline & $\begin{array}{c}\text { FTL Concept } \\
(\mathrm{N}-\text { Days }=4 \text { days })\end{array}$ & & & $X$ & & & $X$ \\
\hline
\end{tabular}

Table 1: Limitations of hole making operations

\begin{tabular}{|c|c|}
\hline Item Classification & S (Days) \\
\hline A & 10 \\
\hline B & 10 \\
\hline C & 15 \\
\hline
\end{tabular}

Table 2, Simulation scenarios

FTL : Full Truckload

LTL: Less than Truckload

\section{Tab. 2: Simulation scenarios}

Thus the following scenarios are:

- Scenario 1: 30 regional distribution centers and with inventory policy (CLS=80\%) and the orders served by LTL strategy.

- Scenario 2: 30 regional distribution centers and with inventory policy (CLS=80\%) and the orders served by FTL strategy ( $\mathrm{N}=2$ days).

- Scenario 3: 30 regional distribution centers and with inventory policy (CLS $=80 \%$ ) and the orders served by FTL strategy ( $\mathrm{N}=4$ days).

- Scenario 4: 30 regional distribution centers and with inventory policy (CLS=90\%) and the orders served by LTL strategy.

- Scenario 5: 30 regional distribution centers and with inventory policy (CLS=90\%) and the orders served by FTL strategy ( $\mathrm{N}=2$ days).

- Scenario 6: 30 regional distribution centers and with inventory policy (CLS=90\%) and the orders served by FTL strategy ( $\mathrm{N}=4$ days).

\subsection{Experiment Results}

Simulation results are otained after running the HSMA for one year. The validation of the model has been accomplished by comparing the simulated results with the historical data provided by the logistic department in the company.

The experiments focus on how significantly the HSMA can explore the effect of the two design factors (inventory \&Transportation) on the selected measures of performance. Four measures of performance are selected to provide measures on the logistics cost and service level.

These measures are the sum of the transportation costs for one year between each warehouse and distribution centre (TTC), the sum of the inventory holding costs for one year for all distribution centres (TIHC), the total logistics cost (TLC), and the total number of unsatisfied immediate (in the same day) customer orders in one year (Service Level).

By the help of Microsoft Excel and Access to assist in scrutinizing the output results, the results are summarized and described in the following diagrams. 


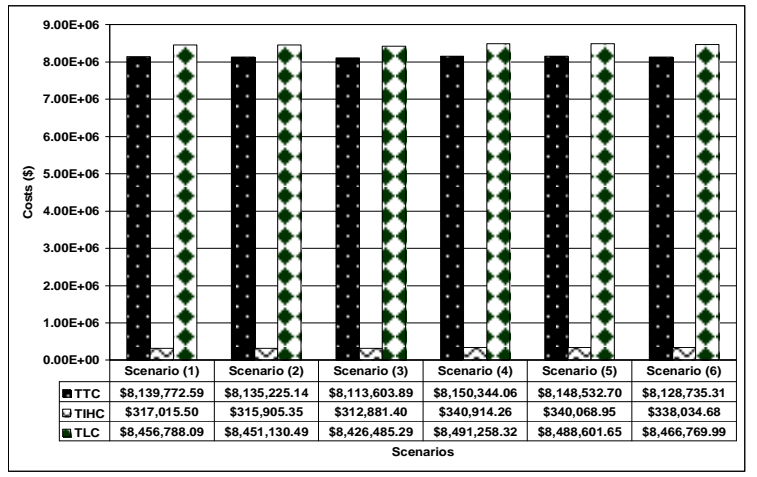

Fig. 3 Total logistics costs for each scenario

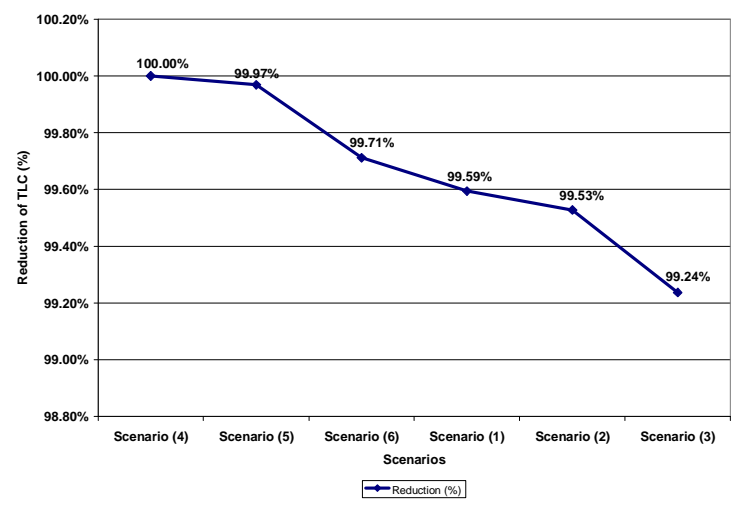

Fig. 4 Reduction percentage for each scenario

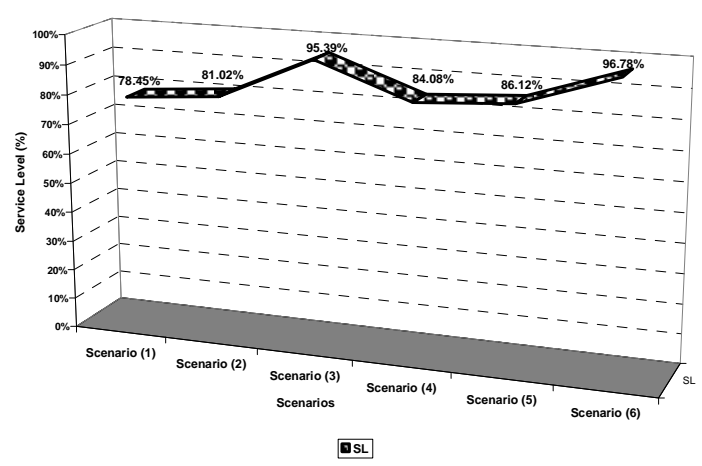

Fig. 5 Service level for each scenario

Referring to the above figures, the following comments can be made based on the results:

- For all scenarios, the four selected measures of performance change significantly as the inventory policies and transportation strategies are altered.

- Scenario (3) and Scenario (6) perform better than the others as in the most of measures of performance, significant improvement in the service level as compared with all scenarios.

- Scenario (3) performs significant reduction in average daily ending inventory.
- N-Days forecasted demand concepts are effective consolidation concepts for designing effective operating distribution strategies.

- Interaction between the reorder point design and the consolidation concepts is remarkable. So more consideration of this interaction is powerful for designing effective operating distribution strategies.

- Distribution strategies with Full Truckload perform better than the other strategies.

- In all six scenarios the 4-Days Forecasted Demand is the optimal strategies for improving system performances, especially in terms of service level.

- On the other hand, a high average daily ending inventory (TIHC) cannot be correlated with the high service levels (SL) in all the strategies.

- Furthermore, as seen in Fig. 4, the percentage of decreasing the total logistics costs by each operating distribution strategy is very small. This can be justified by the highly sharing of transportation costs in the total logistics costs.

\section{CONCLUSIONS}

The distribution operating strategies in the supply chains have a significant impact on the total logistics costs. The strategies that reduce system wide costs and improve the efficiency of the distribution networks should be more evaluated.

In this paper, we presented a hybrid simulation approach combining the analytic and simulation model for a real logistic-distribution system in supply chain environment. The hybrid method uses advantages of both modeling methods while avoiding demerits of both methods. Through experiments of the hybrid approach, it is verified that the initial analytic solutions can not be accepted in the real world system having stochastic characteristics which are not included in analytic model.

Hybrid simulation modeling approach provides more realistic optimal solutions for the integrated distribution (inventory \& transportation) planning in supply chain that are quite different from the initial mathematical and analytic solutions.

Many experiments and analysis have been implemented by this approach. These analyses show and prove that, a hybrid simulation approach is a useful modeling tool for studying and evaluating the efficient of different planning and operating activities in real supply chains.

There are many opportunities for expansion of the hybrid simulation modeling approach presented in this study. The following are the areas where model expansion would be of benefit to future studies: more 
common inventory policies, different cost and network structures, and new trends and a new prescriptive of the Supply Chain

\section{REFERENCES}

[1] Thomas, D.J. and Griffin, P.M., "Coordinated supply chain management," European Journal of Operational Research, Vol. 94, No. 1, 1-15, 1996.

[2] Eliter, T./Subrahmanian, V.S./ Pick, G. "Heterogeneous active agents", Computer Science Technical Report Series, University of Maryland, College Park, 1998.

[3] Swaminathan, J.M., Smith, S.F., and Sadeh, N.M., "Modeling supply chain dynamics: a multiagent approach," Decision Sciences, Vol. 29, No. 3, 607-631, 1998.

[4] Lee, Y.H, Kim, S.H., " Optimal productiondistribution planning in supplychain management using a hybrid simulation-analytic approach" Proceedings of the 2000 Winter Simulation Conference, 2000.

[5] Jain, S., Workman, R.W., Collins, L.M., and Ervin, E.C.,"Development of a high-level supply chain simulation model," Proceedings of the 2001 Winter Simulation Conference, pp. 11291137, 2001.
[6] Díaz, L.,and Buxmann P., "The value of cooperative planning in supply chains, a simulative approach", The 11th European Conference on Information Systems" (ECIS 2003), Naples, Italy, 1-17, 2003.

[7] Chu, E., "A review of simulation studies on supply chain management", Journal, 2003.

[8] Terzi, S. and Cavalieri,S., "Simulation in the supply chain context: a survey", Computers in industry, Vol. 53, No.1, 3-16, 2004.

[9] Kleijnen , J. P.C., "Supply chain simulation tools and techniques: asurvey", International Journal of Simulation \& Process Modelling, Vol. 1, 8289, 2005.

[10] Pundoor, G., "Supply chain simulation models for evaluating the impact of rescheduling frequencies", MS 2002-9, Institute for Systems Research, University of Maryland, 2002.

[11] Banks, J., Carson, J.S., Nelson, B.L. and Nicol, D.M., "Discrete-Event System Simulation", 3rd Edition, Prentice Hall, 2003.

[12]SDZ GmbH, "DOSIMIS-3 manual”, SimulationsDienstleistungsZentrum, Germany, 2004.

[13] Axsäter, S., "Inventory Control”, Kluwer Academic Publishers. Boston, MA, 2000. 